\title{
Adjuvant Chemoradiation for Pancreatic Adenocarcinoma: The Johns Hopkins Hospital-Mayo Clinic Collaborative Study
}

\author{
Charles C. Hsu, MD, PhD ${ }^{1,7}$, Joseph M. Herman, MD, MSc ${ }^{1}$, Michele M. Corsini, $\mathrm{MD}^{2}$, Jordan M. Winter, $\mathrm{MD}^{3}$, \\ Matthew D. Callister, $\mathrm{MD}^{4}$, Michael G. Haddock, $\mathrm{MD}^{2}$, John L. Cameron, $\mathrm{MD}^{3}$, Timothy M. Pawlik, MD, MPH ${ }^{3}$, \\ Richard D. Schulick, $\mathrm{MD}^{3}$, Christopher L. Wolfgang, $\mathrm{MD}, \mathrm{PhD}^{3}$, Daniel A. Laheru, $\mathrm{MD}^{5}$, Michael B. Farnell, $\mathrm{MD}^{6}$, \\ Michael J. Swartz, MD ${ }^{1}$, Leonard L. Gunderson, MD, $\mathrm{MS}^{4}$, and Robert C. Miller, $\mathrm{MD}^{2}$ \\ ${ }^{1}$ Department of Radiation Oncology and Molecular Radiation Sciences, Johns Hopkins Hospital, Baltimore, MD; \\ ${ }^{2}$ Department of Radiation Oncology, Mayo Clinic, Rochester, MN; ${ }^{3}$ Department of Surgery, Johns Hopkins Hospital, \\ Baltimore, MD; ${ }^{4}$ Department of Radiation Oncology, Mayo Clinic Arizona, Scottsdale, AZ; 5 Department of Medicine, \\ Johns Hopkins Hospital, Baltimore, MD; ${ }^{6}$ Department of Surgery, Mayo Clinic, Rochester, MN; ${ }^{7}$ Department of Radiation \\ Oncology, University of California, San Francisco, San Francisco, CA
}

\begin{abstract}
Background. Survival for pancreatic ductal adenocarcinoma is low, the role of adjuvant therapy remains controversial, and recent data suggest adjuvant chemoradiation (CRT) may decrease survival compared with surgery alone. Our goal was to examine efficacy of adjuvant CRT in resected pancreatic adenocarcinoma compared with surgery alone.

Materials and Methods. Patients with pancreatic adenocarcinoma at Johns Hopkins Hospital ( $n=794,1993-2005)$ and Mayo Clinic $(n=478,1985-2005)$ following resection who were observed $(n=509)$ or received adjuvant 5-FU based CRT (median dose 50.4 Gy; $n=583$ ) were included. Cox survival and propensity score analyses assessed associations with overall survival. Matched-pair analysis by treatment group (1:1) based on institution, age, sex, tumor size/stage, differentiation, margin, and node positivity with $N=496(n=248$ per treatment arm) was performed.
\end{abstract}

Presented as Oral Presentations at the 50th annual meeting of the American Society for Therapeutic Radiology and Oncology (ASTRO), Boston, MA 2008 and the Gastrointestinal Cancers Symposium (ASCO/ASTRO/SSO/AGA), Orlando, FL 2008.

(C) The Author(s) 2009. This article is published with open access at Springerlink.com

First Received: 23 May 2009;

Published Online: 20 January 2010

J. M. Herman, MD, MSc

e-mail: jherma15@jhmi.edu
Results. Median survival was 18.8 months. Overall survival (OS) was longer among recipients of CRT versus surgery alone (median survival 21.1 vs. 15.5 months, $P<.001 ; 2$ - and 5-year OS 44.7 vs. $34.6 \% ; 22.3$ vs. $16.1 \%$, $P<.001)$. Compared with surgery alone, adjuvant CRT improved survival in propensity score analysis for all patients by $33 \%(P<.001)$, with improved survival when stratified by age, margin, node, and T-stage $(\mathrm{RR}=0.57$ $0.75, P<.05)$. Matched-pair analysis demonstrated OS was longer with CRT (21.9 vs. 14.3 months median survival; 2and 5 -year OS 45.5 vs. $31.4 \%$; 25.4 vs. $12.2 \%, P<.001$ ). Conclusions. Adjuvant CRT is associated with improved survival after pancreaticoduodenectomy. Adjuvant CRT was not associated with decreased survival in any risk group, even in propensity score and matched-pair analyses. Further studies evaluating adjuvant chemotherapy compared with adjuvant chemoradiation are needed to determine the most effective combination of systemic and local-regional therapy to achieve optimal survival results.

\section{INTRODUCTION}

Annually, approximately 42,000 cases of pancreatic cancer are diagnosed in the United States, with about 35,000 attributable cancer-related deaths each year. ${ }^{1}$ Most patients with pancreatic adenocarcinoma present with advanced disease, precluding curative therapy. While surgical resection is the only curative modality for those with localized disease, only $10 \%-15 \%$ of pancreatic adenocarcinoma patients are resectable at presentation. ${ }^{2-5}$ 
Specifically, 5-year survival following pancreaticoduodenectomy (PD) with curative intent is less than $20 \%{ }^{4-6}$ Both local and systemic relapses are common following PD or total pancreatectomy, suggesting systemic and local adjuvant therapy are necessary to improve outcomes. ${ }^{7}$

In an effort to improve both local control and overall survival following resection, the efficacy of adjuvant chemoradiation (CRT) and chemotherapy have been evaluated in several trials. The Gastrointestinal Tumor Study Group (GITSG) findings support the use of adjuvant CRT, while the European Organisation for Research and Treatment of Cancer (EORTC) trials suggested benefit. ${ }^{8-10}$ However, clinical trial outcomes from the European Study Group for Pancreatic Cancer (ESPAC-1) suggested a detrimental effect on survival compared with adjuvant chemotherapy or surgery alone. ${ }^{11,12}$ Despite many concerns raised regarding study design, radiation administration, and quality control of the study, ${ }^{13-18}$ conclusions drawn from the ESPAC-1 trial have raised doubts about the implementation of adjuvant CRT for pancreatic adenocarcinoma in spite of other data showing efficacy of adjuvant or neoadjuvant CRT for other gastrointestinal cancer sites. ${ }^{19,20}$ Recently, several single institution retrospective and prospective studies have demonstrated the utility of adjuvant CRT compared with surgery alone, with adjuvant CRT outcomes similar to those of a recent randomized trial evaluating gemcitabine versus fluorouracil chemotherapy for adjuvant CRT. ${ }^{21-24}$ However, compared with randomized trials, retrospective studies frequently are plagued with concerns of bias associated with the nonrandom allocation of patients to adjuvant treatment modalities or observation. ${ }^{25}$

The goal of this study was to combine the experiences of two major academic institutions that treat high volumes of patients with pancreatic adenocarcinoma to examine the efficacy of adjuvant CRT. This study proposed to: (1) determine factors that predict survival following PD, (2) examine the benefit of adjuvant CRT by risk group, and (3) account for biases associated with treatment selection of adjuvant CRT in retrospective data through propensity score analysis and matched-pair analysis.

\section{METHODS}

\section{Study Design and Participants}

This study was approved by the institutional review boards of the Mayo Clinic, Rochester, and the Johns Hopkins Hospital. Study participants were drawn from all patients who underwent pancreaticoduodenectomy (PD) for pancreatic cancer at the Johns Hopkins Hospital between 1993 and 2005 ( $n=794$, prospectively collected) and the Mayo Clinic, Rochester, from 1985 to 2005 ( $n=592$, retrospectively collected). Of the 1386 patients, individuals were excluded who died within 60 days of surgery, had unresectable disease (T4 or M1), periampullary tumors, received intraoperative radiation therapy or experimental pancreatic cancer vaccine therapy, had distal pancreatectomies, single modality adjuvant treatment, or were missing data on margin status, nodal status, or histologic grade $(n=294)$.

\section{Surgery}

Patients underwent either a pylorus-preserving, classic, or total PD. A pylorus-preserving PD included resection of the head and uncinate process of the pancreas, distal bile duct, all but the most proximal duodenum, and gallbladder, when present. A classic PD also included the antrum of the stomach. A total PD included the entire pancreas with the spleen. Lymph nodes were considered positive if any lymph node in the resection specimen contained metastatic carcinoma, whether it was involved by direct extension or was contiguous with the primary tumor. Resection margins were considered positive if the carcinoma was close (within $1 \mathrm{~mm}$ ) or present at the final pancreatic neck, uncinate process, bile duct, duodenal, or retroperitoneal soft tissue margin. All pathology specimens were reviewed by either a single pathologist at JHH or centrally at the Mayo Clinic. Variables included in analyses for which we had information for all study participants were age, gender, surgery type, year of surgery, surgical margin, nodal status, and histologic grade. T-stage was unknown for $16.6 \%$ $(n=181)$ of participants, all from JHH. Race, comorbid diseases (diabetes, cardiovascular disease, chronic obstructive pulmonary disease, hypertension), and operative characteristics (surgical complications, vascular invasion, perineural invasion) were available for a significant portion of patients from JHH but not the Mayo Clinic and were not included in analyses. Patient follow-up information was obtained from hard-copy and electronic hospital charts. Survival was determined and cross checked by review of clinical follow-up information, cancer center abstracting services, or the Social Security Death Index.

\section{Adjuvant CRT}

Of the 1,092 patients who underwent resection with curative intent and met the inclusion criteria, 56.6\% $(n=618)$ were from $\mathrm{JHH}$ and $43.4 \%(n=474)$ from the Mayo Clinic. Median follow-up for the total study was 18.2 months. Of the 1,092 patients, $509(46.6 \%)$ received surgery alone, without any adjuvant therapy. When stratified by adjuvant treatment, survival did not significantly differ by institution $(P>.35$, results not shown). Patients who underwent PD with adjuvant CRT $(n=583)$ received 5-fluorouracil (FU) based adjuvant CT, with $98 \%$ of Mayo 
Clinic adjuvant CRT patients receiving concurrent 5-FU based CT while JHH patients were offered continuous infusion FU with RT followed by maintenance FU for an additional 2-6 months. Of adjuvant CRT patients who underwent $\mathrm{PD}$ at $\mathrm{JHH}, 50.4 \%$ received adjuvant therapy at JHH and $49.6 \%$ were treated elsewhere, with similar recommendations for CRT prior to discharge. Of adjuvant CRT patients who underwent resection at the Mayo Clinic, $51.5 \%$ received adjuvant CRT at the Mayo Clinic and $48.5 \%$ elsewhere. There was no statistically significant difference in survival between the four groups who underwent PD at JHH and received adjuvant $\mathrm{CRT}$ at $\mathrm{JHH}, \mathrm{PD}$ at $\mathrm{JHH}$ and adjuvant CRT at an outside facility, PD at Mayo Clinic and adjuvant CRT at the Mayo Clinic, and PD at Mayo Clinic and adjuvant CRT at an outside facility $(P=.35)$. The details of adjuvant radiotherapy have been described in detail elsewhere. ${ }^{22,23}$ In brief, adjuvant external-beam RT was delivered with linear accelerators using multiple-field techniques, with the median RT dose of $50.4 \mathrm{~Gy}$ and daily fraction of $1.8 \mathrm{~Gy}$. The majority of patients received a continuous course of radiation therapy without a planned break.

\section{Statistical Analysis}

Statistical analyses were performed using STATA, version 9 (Stata, College Station, TX). Summary statistics for continuous and dichotomous variables are provided. Tests of differences were performed using $t$ tests and $\chi^{2}$ tests. For analyses stratified by $\mathrm{T}$ stage, $\chi^{2}$ tests and analyses were performed including only those with known status, as indicated. The primary outcome variable was OS, defined as the time from surgical resection for pancreatic cancer to death, as described previously. ${ }^{22,23}$ Survival curves were estimated using Kaplan-Meier techniques. ${ }^{26}$ Comparisons of OS between groups were made using the log-rank test. Median OS (in months) with $95 \%$ confidence intervals $(95 \% \mathrm{CI})$ was estimated within each risk group and by adjuvant treatment. The proportion of individuals surviving up to 2 and 5 years was calculated using life tables, with comparison by adjuvant treatment performed using the log-rank test with survival time censored at 2 and 5 years, respectively.

Proportional hazards models were used to examine the association with mortality of adjuvant treatment and other patient characteristics. ${ }^{27}$ Univariate analyses were used to examine individual risk factors and associations with mortality. To examine the independent association of adjuvant therapy and OS after surgical resection, multivariate analyses were performed adjusting for confounders, including age $\geq 70$ (yes vs. no), sex, institution, margin positivity, node positivity, tumor differentiation (G1/2 vs. G3/4), surgery type, and T-stage. Data on postoperative recovery, performance status, and CA 19-9 were either unavailable or insufficient, not allowing for informative analyses. Among all patients, T-stage was missing from $181 \mathrm{JHH}$ patients. Multivariate models that included $(n=1,092)$ and excluded $(n=911)$ those with missing T-stage were comparable (results not shown), and these individuals were included in our final analyses. To further examine the association of mortality with adjuvant CRT compared with surgery alone, we also stratified by risk factors associated with mortality.

To account for biases in treatment effect arising from nonrandom allocation of patients to treatment groups, propensity score analyses were performed. ${ }^{28} \mathrm{~A}$ propensity score was generated using 16 variables, including age, gender, surgery type, year of surgery, surgical margin, node status, histologic grade, T-stage, race, diabetes, cardiovascular disease, chronic obstructive pulmonary disease, hypertension, surgical complications, vascular invasion, and perineural invasion of the tumor. Those missing information for variables were coded as a separate category such that a propensity score was generated for all participants. Propensity score analysis required calculation of the conditional probabilities for the two treatment groups (adjuvant CRT vs. surgery alone) using multivariate regression, generating a propensity score, which was used in multivariate Cox regression model. ${ }^{28}$ For the propensity score, the receiver operating curve area under the curve $=0.74$, and $68.4 \%$ of patients were correctly classified to treatment according to the multivariate model.

To further account for treatment selection biases associated with retrospective data, 1-to-1 matching by treatment group was performed (adjuvant CRT and surgery alone). Individuals were matched on institution, age group, sex, surgery type, tumor size $(\mathrm{JHH})$, tumor stage (Mayo Clinic), tumor differentiation, margin status, and nodal status. In total, there were 496 patients in this analysis ( $n=248$ adjuvant CRT and $n=248$ surgery alone). Of this group, 228 were from Hopkins and 268 from Mayo Clinic, Rochester. Individuals did not differ significantly by treatment group for any of the matched characteristics. Unadjusted and stratified Cox proportional hazards analyses were performed.

\section{RESULTS}

\section{Clinical Characteristics of Study Population, by Adjuvant Treatment Group}

As shown in Table 1, compared with those who underwent surgery without adjuvant therapy, patients who received adjuvant CRT were younger at time of treatment (median age 70.2 vs. 64.7 years, $P<.001$ ), had worse histology (grade 3 or $4: 51 \%$ vs. $59 \%, P=.019$ ), and had more margin positive disease $(31 \%$ vs. $35 \%, P<.001)$. 
TABLE 1 Baseline characteristics

\begin{tabular}{|c|c|c|c|}
\hline & $\begin{array}{l}\text { Observation } \\
\text { only } \\
(n=509)\end{array}$ & $\begin{array}{l}\text { Adjuvant } \\
\text { chemoradiation } \\
\text { therapy } \\
(n=583)\end{array}$ & $P$ value \\
\hline \multicolumn{4}{|l|}{ Demographic } \\
\hline \multicolumn{4}{|l|}{ Age at surgery (year) } \\
\hline Mean (SD) & $68.9(10.9)$ & $63.7(3.1)$ & $<.001$ \\
\hline Median (Range) & $70.2(34-92)$ & $\begin{array}{l}64.7 \\
(29.4-85.6)\end{array}$ & \\
\hline \multicolumn{4}{|l|}{ Gender } \\
\hline Male, no. $(\%)$ & $260(51.1)$ & $316(54.2)$ & .303 \\
\hline \multicolumn{4}{|l|}{ Treatment } \\
\hline \multicolumn{4}{|l|}{ Institution } \\
\hline $\begin{array}{l}\text { The Johns Hopkins } \\
\text { Hospital }\end{array}$ & $346(68.0)$ & $272(46.7)$ & $<.001$ \\
\hline $\begin{array}{l}\text { The Mayo Clinic } \\
\text { Rochester }\end{array}$ & $163(32.0)$ & $311(53.3)$ & \\
\hline \multicolumn{4}{|l|}{ Surgery type } \\
\hline Classic PD & $196(38.5)$ & $247(42.4)$ & .307 \\
\hline Pylorus-preserving PD & $279(54.8)$ & $306(52.5)$ & \\
\hline $\begin{array}{l}\text { Classic/PP total } \\
\text { pancreatectomy }\end{array}$ & $34(6.7)$ & $30(5.1)$ & \\
\hline \multicolumn{4}{|l|}{ Tumor characteristics } \\
\hline \multicolumn{4}{|l|}{ Primary tumor } \\
\hline $\mathrm{T} 1$ & $35(6.9)$ & $33(5.7)$ & $.239^{\mathrm{a}}$ \\
\hline $\mathrm{T} 2$ & $79(15.5)$ & $107(18.4)$ & \\
\hline $\mathrm{T} 3$ & $322(63.3)$ & $335(57.5)$ & \\
\hline Unknown & $73(14.3)$ & $108(18.5)$ & \\
\hline \multicolumn{4}{|l|}{ Nodal status } \\
\hline No & $163(32.0)$ & $191(32.8)$ & .795 \\
\hline $\mathrm{N}+$ & $346(68.0)$ & $392(67.2)$ & \\
\hline \multicolumn{4}{|l|}{ Histologic grading } \\
\hline 1 & $12(2.4)$ & $18(3.1)$ & .027 \\
\hline 2 & $236(46.4)$ & $223(38.3)$ & \\
\hline 3 & $239(47.0)$ & $302(51.8)$ & \\
\hline 4 & $22(4.3)$ & $40(6.9)$ & \\
\hline \multicolumn{4}{|l|}{ Margin } \\
\hline Negative & $349(68.6)$ & $380(65.2)$ & $<.001$ \\
\hline Positive & $160(31.4)$ & $203(34.8)$ & \\
\hline
\end{tabular}

$P D$ pancreaticoduodenectomy, $P P$ pylorus preserving

${ }^{a}$ Chi-square compares only non-missing values, with T-stage unknown among $n=181 \mathrm{JHH}$ patients

Gender, surgery type, tumor stage, and nodal status were not associated with the type of adjuvant treatment.

\section{Clinical and Treatment Characteristics Associated with Overall Mortality}

In the 1,092 patients in the study population, median follow-up was 18.2 months. Median overall survival was
18.8 months (95\% CI: 17.7-20.0), with 2-year and 5-year overall survival of 40.0 and $19.4 \%$. As shown in Table 2, those who were $\geq 70$ years had a $20 \%$ increase in mortality compared with those $<70$ years of age (median OS 17.5 vs. 19.7 months, $P=.008$ ). T3 stage, poor histological differentiation, node positivity, and margin positivity each increased risk of death by approximately $45 \%$ compared with those without the respective risk characteristic (RR range 1.44-1.47, all $P<.001$ ). Gender and surgery type were not associated with mortality differences. As illustrated in Fig. 1, compared with those who underwent surgery alone, adjuvant CRT improved survival with a RR of 0.73 (95\% CI: 0.64-0.84) with longer median OS (15.5 vs. 21.1 months) and greater 2-year (34.6 vs. $44.7 \%)$ and 5year survival $(16.1$ vs. $22.3 \%)$, respectively $(P<.001)$.

\section{Associations of Adjuvant CRT with Overall Mortality, Overall, and by Risk Group}

In Table 3, adjustments were made for known and potential confounders, including age, gender, institution, surgery type, tumor stage, margin status, node status, and tumor differentiation, and a propensity score was included in the multivariate model to further adjust for potential bias associated with treatment selection. The results demonstrated that compared with surgery alone, adjuvant CRT significantly decreased risk of mortality in the study population with an adjusted RR of 0.67 (95\% CI: 0.58-0.78, $P<.001$, Table 3). Data were further stratified by known clinical predictors of mortality, and among each risk group, adjuvant CRT was protective (adjusted RR range: 0.560.83 ) and statistically significant in all groups, except those with tumor stage 1-2 (adjusted RR 0.83, 95\% CI: 0.66$1.03, P=.087)$.

\section{Matched-Pair Analysis}

To further attempt to account for treatment selection biases associated with retrospective data, one-to-one matching was performed by treatment group, on the variables of institution, age, sex, tumor size or stage, histologic differentiation, margin status, and nodal status, resulting in a total of 496 patients with 248 per treatment arm, either surgery alone or surgery with adjuvant CRT. There were no statistically significant differences $(P>.05)$ among matched variables by treatment group (results not shown). As seen in Fig. 2, matched-pair analysis demonstrated overall survival was improved with adjuvant CRT versus surgery alone, with 21.9 vs. 14.3 month median OS and better 2year $(45.5 \%$ vs. $31.4 \%)$ and 5-year survival (25.4 vs. 12.2\%). Adjuvant CRT decreased risk with RR 0.59 (95\% CI: $0.48-0.72, P<.001)$. As shown in Fig. 3 , adjuvant CRT compared with surgery alone was associated with 
TABLE 2 Associations with overall survival and patient, tumor, and treatment characteristics

\begin{tabular}{|c|c|c|c|c|c|c|}
\hline & No. (\%) & Median surv, months & 2-year OS, \% & 5-year OS, \% & Univariate RR $(95 \% \mathrm{CI})$ & $P$ value \\
\hline \multicolumn{7}{|l|}{ Demographic } \\
\hline \multicolumn{7}{|l|}{ Age (year) } \\
\hline$<70$ & $645(59.1)$ & 19.7 & 42.1 & 21.6 & 1.00 & \\
\hline$\geq 70$ & $447(40.9)$ & 17.5 & 37.1 & 16.2 & $1.20(1.05-1.37)$ & .008 \\
\hline \multicolumn{7}{|l|}{ Gender } \\
\hline Female & $516(47.3)$ & 19.5 & 40.9 & 18.3 & 1.00 & \\
\hline Male & $576(52.8)$ & 18.5 & 39.2 & 20.5 & $0.98(0.85-1.12)$ & .730 \\
\hline \multicolumn{7}{|l|}{ Treatment } \\
\hline \multicolumn{7}{|l|}{ Adjuvant treatment } \\
\hline None & $509(46.6)$ & 15.5 & 34.6 & 16.1 & 1.00 & \\
\hline Adjuvant CRT & $583(53.4)$ & 21.1 & 44.7 & 22.3 & $0.73(0.64-0.84)$ & $<.001$ \\
\hline \multicolumn{7}{|l|}{ Surgery type } \\
\hline Class PD & $443(40.6)$ & 19.7 & 40.2 & 22.5 & 1.00 & \\
\hline PPPD & $585(53.6)$ & 18.5 & 40.2 & 17.1 & $1.13(0.98-1.30)$ & .093 \\
\hline Classic/PP total panc & $64(5.9)$ & 14.9 & 36.5 & 18.3 & $1.19(0.89-1.60)$ & .228 \\
\hline \multicolumn{7}{|l|}{ Tumor characteristics } \\
\hline \multicolumn{7}{|l|}{ Primary tumor $^{\mathrm{a}}$} \\
\hline $\mathrm{T} 1-2$ & $254(27.9)$ & 26.0 & 52.0 & 29.2 & 1.00 & \\
\hline $\mathrm{T} 3$ & $657(72.1)$ & 17.7 & 36.5 & 16.9 & $1.44(1.21-1.70)$ & $<.001$ \\
\hline \multicolumn{7}{|l|}{ Nodal status } \\
\hline $\mathrm{N}-$ & $354(32.4)$ & 24.8 & 50.8 & 26.0 & 1.00 & \\
\hline $\mathrm{N}+$ & $738(67.6)$ & 17.4 & 34.9 & 15.9 & $1.46(1.26-1.69)$ & $<.001$ \\
\hline \multicolumn{7}{|l|}{ Histologic grading } \\
\hline $\mathrm{G} 1 / 2$ & $489(44.8)$ & 23.3 & 49.1 & 23.5 & 1.00 & \\
\hline $\mathrm{G} 3 / 4$ & $603(55.2)$ & 15.7 & 32.5 & 16.1 & $1.44(1.26-1.65)$ & $<.001$ \\
\hline \multicolumn{7}{|l|}{ Margin } \\
\hline Negative & $729(66.8)$ & 21.0 & 45.3 & 22.9 & 1.00 & \\
\hline Positive & $363(33.2)$ & 15.1 & 29.5 & 12.7 & $1.47(1.28-1.69)$ & $<.001$ \\
\hline
\end{tabular}

$P D$ pancreaticoduodenectomy, $P P$ pylorus preserving, Total panc total pancreatectomy, $N-$ node negative, $N+$ node positive, Surv survival, mo months, $O S$ overall survival

${ }^{\text {a }}$ T-stage missing $(n=181)$ only among JHH patients

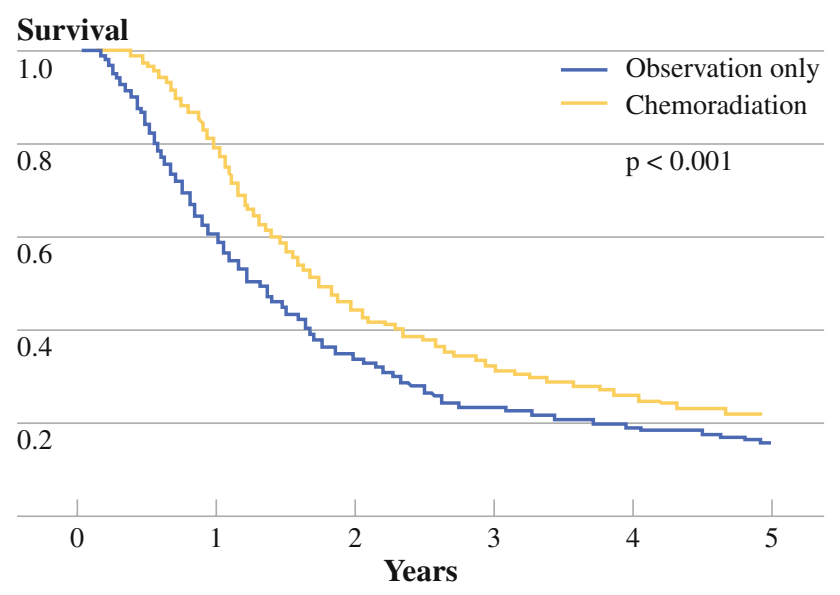

FIG. 1 Kaplan-Meier plot of overall survival among all 1,092 resected pancreatic adenocarcinoma patients with (yellow line) and without (blue line) adjuvant chemoradiation therapy $(P<.001)$ improved survival among both margin negative (RR 0.61, 95\% CI: $0.47-0.77, P<.001)$ and margin positive (RR 0.52 , 95\% CI: $0.36-0.74, P<.001)$ disease. Improved survival with adjuvant CRT was also seen among node positive disease (RR 0.53, 95\% CI: $0.42-0.68, P<.001$ ) and bordered on statistical significance among node negative disease (RR $0.71,95 \% \mathrm{CI}$ : $0.49-1.02, P=.063$ ). A similarly protective association of adjuvant CRT with overall survival was seen when stratified by T-stage and by tumor differentiation (results not shown).

\section{DISCUSSION}

At two high-volume centers for treatment of pancreatic adenocarcinoma, adjuvant CRT was significantly associated with improved survival after pancreaticoduodenectomy compared with surgery alone, regardless of age, tumor size, 


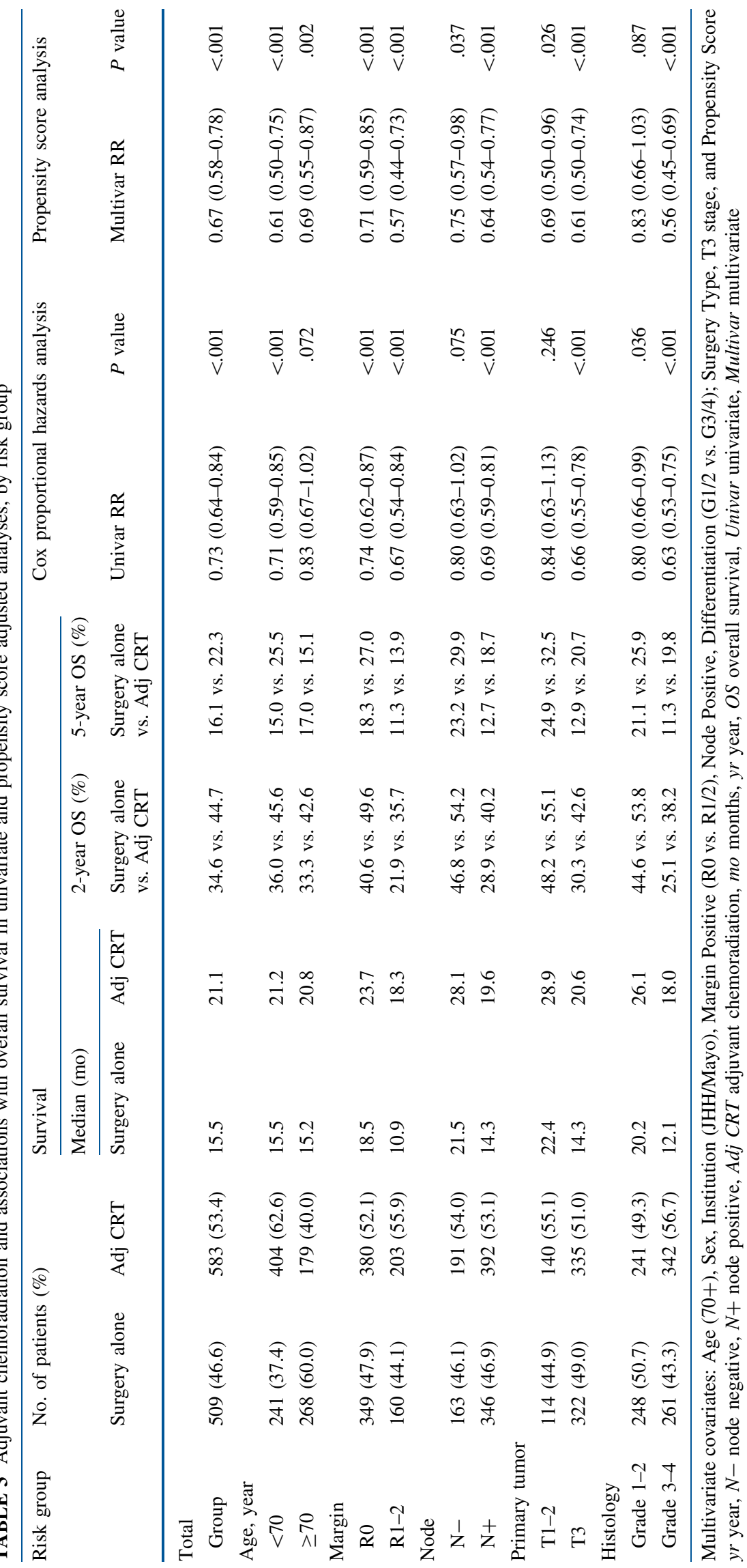




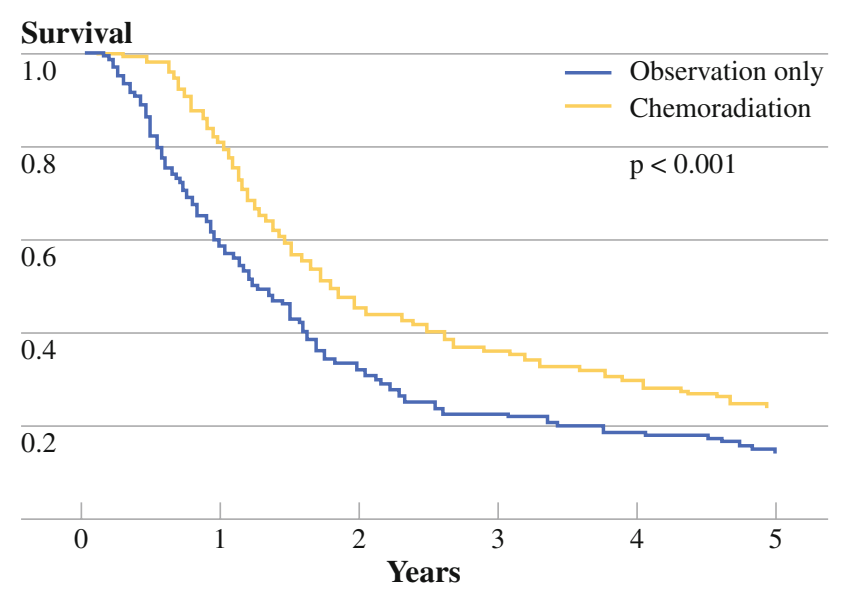

FIG. 2 Matched-pair analysis: Kaplan-Meier plot of overall survival of 1:1 matched 496 resected pancreatic adenocarcinoma patients with ( $n=248$, yellow line $)$ and without $(n=248$, blue line $)$ adjuvant chemoradiation therapy $(P<.001)$

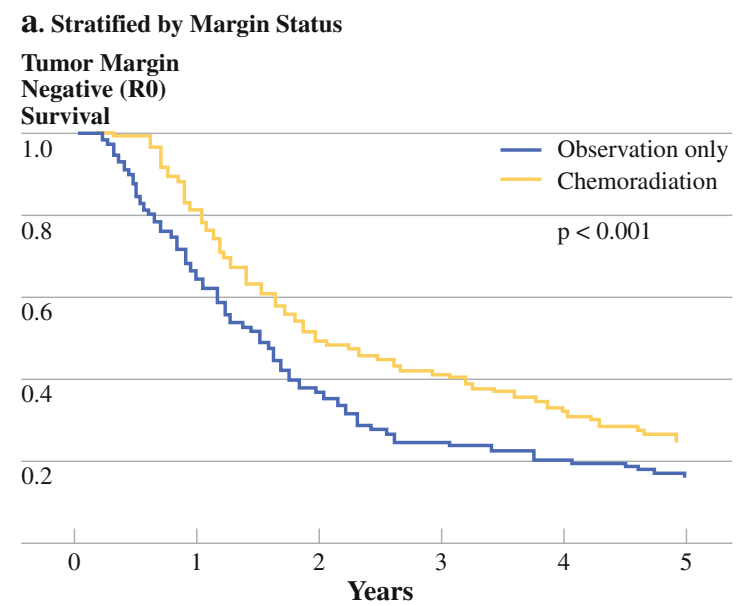

b. Stratified by Node Status

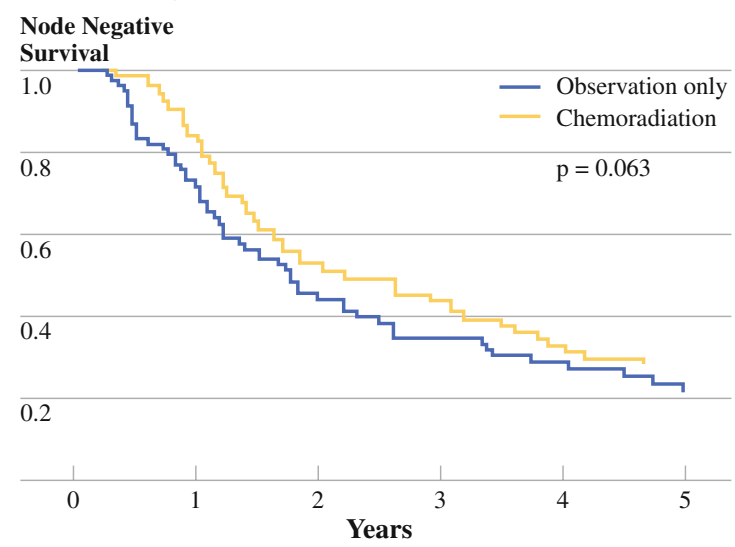

FIG. 3 Stratified Matched-Pair Analysis. a Kaplan-Meier plot of overall survival of 1:1 matched resected pancreatic adenocarcinoma patients with (yellow line) and without (blue line) adjuvant chemoradiation therapy, stratified by margin negative (left, $n=358$, $P<.001)$ and margin positive (right, $n=138, P<.001)$ status. $\mathbf{b}$ margin status, or node status. After adjustment for confounders in the propensity score analysis, adjuvant CRT improved overall survival by approximately $33 \%$ $(P<.001)$, with improved median (15.5 vs. 21.1 months), 2-year (34.6 vs. $44.7 \%$ ) and 5-year (16.1 vs. 22.3\%) overall survival $(P<.001)$. Furthermore, risk of mortality was consistently decreased among all risk-stratified subgroups (range RR 0.56-0.83) with the addition of adjuvant CRT. The effects of adjuvant CRT appeared to improve survival regardless of resection or tumor status, including patients who were margin positive (adjusted HR 0.57, $P<.001$ ), margin negative (adjusted HR $0.71, P<.001$ ), node positive (adjusted HR 0.64, $P<.001$ ), or node negative (adjusted HR $0.75, P<.037)$.

For patients with resectable pancreatic adenocarcinoma, the recommendation of adjuvant CRT in the United States has been largely based on results of the GITSG, which demonstrated improved survival for those who underwent
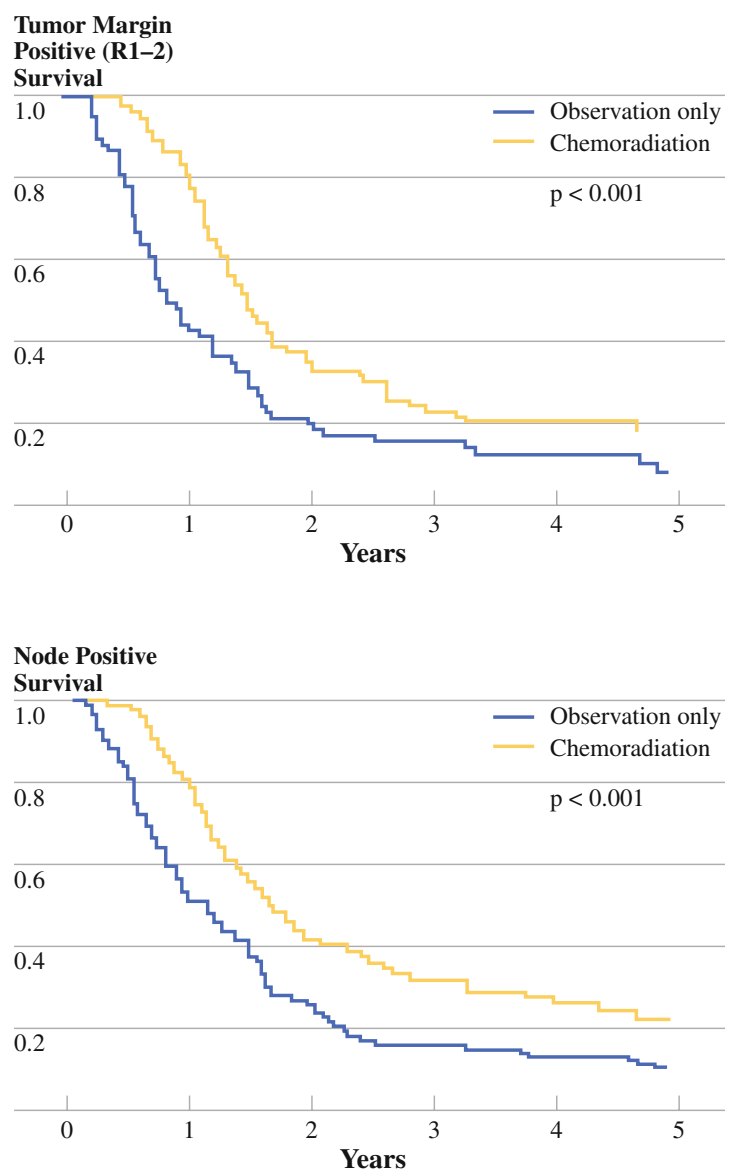

Kaplan-Meier plot of overall survival of 1:1 matched resected pancreatic adenocarcinoma patients with (yellow line) and without (blue line) adjuvant chemoradiation therapy, stratified by node negative (left, $n=160, P=.063$ ) and node positive (right, $n=336, P<.001)$ status 
adjuvant CRT compared with surgery alone (median OS 10.9 vs. 21.0 months, $P=.04){ }^{8}$ These results were further confirmed in an additional 30 patients nonrandomly assigned to adjuvant CRT. ${ }^{29}$ However, the GITSG study has been criticized for its small sample size $(n=43)$ and obsolete use of split-course radiation therapy.

In line with GITSG, the EORTC phase III trial found that compared with those who underwent surgery alone $(n=54)$, those who received adjuvant CRT $(n=60)$ had an improvement in median overall survival (17.1 vs. 12.6 months), but it only approached statistical significance $(P=.099) .{ }^{8}$ However, a reanalysis using a one-sided logrank test suggested statistical significance $(P=.049)$ for 2 year overall survival. ${ }^{10}$

More recently, the ESPAC-1 study suggested that adjuvant radiation therapy is detrimental to overall survival compared with surgery alone. ${ }^{11,12}$ However, the study's use of several concurrent trials, the option for physicians to deliver background adjuvant therapy prior to randomization, a complex $2 \times 2$ factorial design, lack of central review, and the lack of radiation-field design parameters are a few of the criticisms that question the validity of the findings of ESPAC-1. ${ }^{13-18}$ Though the authors suggest the detriment of adjuvant CRT, the OS results for this treatment arm in the ESPAC-1 study are much poorer than and inconsistent with previous findings from other randomized trials. ${ }^{8,15,24}$ This collaborative study pools data from the Johns Hopkins Hospital and Mayo Clinic, Rochester, and demonstrates that adjuvant CRT is not detrimental compared with surgery alone and appears to offer significant benefit for overall survival.

The results in the current study are consistent with the GITSG and EORTC randomized trials and also confirm the results of several single institution studies as well as a national surveillance study. ${ }^{3,21-23}$ Additionally, adjuvant CRT outcomes in the current study are similar to a recent U.S. Gastrointestinal Intergroup phase III randomized trial evaluating adjuvant CRT plus gemcitabine versus fluorouracil systemic chemotherapy, with median OS of 20.5 vs. 16.9 months, respectively. ${ }^{24}$

In the current analysis, patients across all risk groups demonstrated improved survival with adjuvant CRT, compared with surgery alone. In no subgroup was there evidence that adjuvant CRT was detrimental. However, there are several limitations of our study. Despite its size combined from the experience of two institutions, we are unable to answer the question of whether adjuvant CRT confers benefit beyond adjuvant chemotherapy. Additionally, our findings are limited by the fact that they are retrospective. Though we attempt to deal with biases associated with treatment selection with both the propensity score and matched-pair analyses, we are limited by the fact that data on performance status, length of hospital stay, comorbid diseases, and postoperative recovery were not available for all patients. These factors may play a significant role in the decision for patients to undergo adjuvant CRT. Additionally, data on surgical complications were only available for the Johns Hopkins patients, and these data were not used as criteria for the matched-pair analysis. It is not possible given these limitations to completely remove the influence of selection bias from our analyses. Furthermore, we do not have data on local recurrence rates since many did not have a prospective plan for follow-up imaging. Nevertheless, we attempt to deal with these issues in as comprehensive a fashion as possible, given our data, and demonstrate the consistency of the benefit of adjuvant CRT on overall survival across risk groups. Furthermore, this study population from two high-volume treatment centers for pancreatic adenocarcinoma, with sufficient surgical, medical oncology, and radiation oncology experience, allowed performance of stratified analyses and more sophisticated statistical analyses that require larger numbers of patients.

The current collaborative analyses attempted to account for treatment selection biases associated with retrospective data through not only propensity score analyses but also matched-pair analyses. Both methods confirmed the association with improved survival of adjuvant CRT over surgery alone (matched-pair HR 0.59, 95\% CI: 0.48-0.72, $P<.001)$.

In summary, this collaborative study from the Johns Hopkins Hospital and Mayo Clinic strongly supports the use of adjuvant CRT in patients with resected pancreatic adenocarcinoma. The benefit is consistent across all highrisk groups, and the status of resection should not alter adjuvant CRT recommendations. These current results, combining the experience of two high-volume institutions, call into question the results of ESPAC-1, which suggested a detrimental effect of adjuvant CRT.

Future studies are needed that focus on the sequencing of treatment modalities and explore aggressive systemic therapies. With regard to sequencing of trimodality treatment, preoperative CRT may be preferable to postoperative CRT for patients in whom R1 and R2 resections would be expected based on preoperative imaging (i.e., borderline or unresectable disease) in an attempt to prevent local and distant recurrence. Neoadjuvant treatment may be preferable as it is unlikely that any adjuvant therapy, including radiation, can successfully sterilize macroscopic residual disease. Some institutions even prefer preoperative CRT for resectable cancers, although it is still unclear which patients are more likely to benefit from preoperative treatment as opposed to upfront surgical resection. ${ }^{30,31}$

With regard to the use of more effective systemic therapy, results now exist from at least two phase III trials that demonstrate a benefit with adjuvant chemotherapy 
over surgery alone for patients with resected pancreas cancer (adjuvant 5FU-Leucovorin in the ESPAC-1 trial and adjuvant gemcitabine in the CONKO-001 trial). ${ }^{11,12,32}$ However, it is unclear whether adjuvant chemotherapy alone has any impact on local recurrence. Although adjuvant CRT appears to improve both local control and survival when compared with surgery alone in select phase II and phase III trials, OS is still inadequate in view of the high rate of hematologic and peritoneal relapse. Accordingly, more effective systemic therapy combined with the most effective local-regional treatment (surgery plus adjuvant CRT) will be needed to achieve optimal survival results.

ACKNOWLEDGMENT We are indebted to the staff and patients of both the Johns Hopkins Hospital and Mayo Clinic for their important contributions.

OPEN ACCESS This article is distributed under the terms of the Creative Commons Attribution Noncommercial License which permits any noncommercial use, distribution, and reproduction in any medium, provided the original author(s) and source are credited.

\section{REFERENCES}

1. Jemal A, Siegel R, Ward E, Hao Y, Xu J, Thun MJ. Cancer statistics, 2009. CA Cancer J Clin. 2009;59:225-49.

2. Cameron JL, Riall TS, Coleman J, Belcher KA. One thousand consecutive pancreaticoduodenectomies. Ann Surg. 2006;244:10-5.

3. Lim JE, Chien MW, Earle CC. Prognostic factors following curative resection for pancreatic adenocarcinoma: a populationbased, linked database analysis of 396 patients. Ann Surg. 2003;237:74-85.

4. Trede M, Schwall G, Saeger HD. Survival after pancreatoduodenectomy. 118 consecutive resections without an operative mortality. Ann Surg. 1990;211:447-58.

5. Geer RJ, Brennan MF. Prognostic indicators for survival after resection of pancreatic adenocarcinoma. Am $J$ Surg. 1993;165:68-72.

6. Crist DW, Sitzmann JV, Cameron JL. Improved hospital morbidity, mortality, and survival after the Whipple procedure. Ann Surg. 1987;206:358-65.

7. Foo ML, Gunderson LL, Nagorney DM, McLlrath DC, van Heerden JA, Robinow JS, et al. Patterns of failure in grossly resected pancreatic ductal adenocarcinoma treated with adjuvant irradiation \pm 5 fluorouracil. Int $J$ Radiat Oncol Biol Phys. 1993;26:483-489.

8. Kalser MH, Ellenberg SS. Pancreatic cancer. Adjuvant combined radiation and chemotherapy following curative resection. Arch Surg. 1985;120:899-903.

9. Klinkenbijl JH, Jeekel J, Sahmoud T, van Pel R, Couvreur ML, Veenhof $\mathrm{CH}$, et al. Adjuvant radiotherapy and 5-fluorouracil after curative resection of cancer of the pancreas and periampullary region: phase III trial of the EORTC gastrointestinal tract cancer cooperative group. Ann Surg. 1999;230:776-82.

10. Garofalo MC, Regine WF, Tan MT. On statistical reanalysis, the EORTC trial is a positive trial for adjuvant chemoradiation in pancreatic cancer. Ann Surg. 2006;244:332-333.

11. Neoptolemos JP, Stocken DD, Friess H, Bassi C, Dunn JA, Hickey $\mathrm{H}$, et al. A randomized trial of chemoradiotherapy and chemotherapy after resection of pancreatic cancer. $N$ Engl J Med. 2004;350:1200-10.

12. Neoptolemos JP, Dunn JA, Stocken DD, Almond J, Link K, Beger $\mathrm{H}$, et al. Adjuvant chemoradiotherapy and chemotherapy in resectable pancreatic cancer: a randomised controlled trial. Lancet. 2001;358:1576-85.

13. Garofalo M, Flannery T, Regine W. The case for adjuvant chemoradiation for pancreatic cancer. Best Pract Res Clin Gastroenterol. 2006;20:403-16.

14. Koshy MC, Landry JC, Cavanaugh SX, Fuller CD, Willett CG, Abrams RA, et al. A challenge to the therapeutic nihilism of ESPAC-1. Int J Radiat Oncol Biol Phys. 2005;61:965-6.

15. Crane CH, Ben-Josef E, Small W, Jr., Morris SL, Beasley M, Leslie M, Bydder S, Spry N. Chemotherapy for pancreatic cancer. N Engl J Med. 2004;350:2713-5.

16. Abrams RA, Lillemoe KD, Piantadosi S. Continuing controversy over adjuvant therapy of pancreatic cancer. Lancet. 2001;358:1565-6.

17. Evans DB, Hess KR, Pisters PW. ESPAC-1 trial of adjuvant therapy for resectable adenocarcinoma of the pancreas. Ann Surg. 2002;236:694-6.

18. Choti MA. Adjuvant therapy for pancreatic cancer-the debate continues. N Engl J Med. 2004;350:1249-51.

19. Macdonald JS, Smalley SR, Benedetti J, Hundahl SA, Estes NC, Stemmermann GN, et al. Chemoradiotherapy after surgery compared with surgery alone for adenocarcinoma of the stomach or gastroesophageal junction. N Engl J Med. 2001;345:725-30.

20. Kapiteijn E, Marijnen CA, Nagtegaal ID, Putter H, Steup WH, Wiggers $\mathrm{T}$, et al. Preoperative radiotherapy combined with total mesorectal excision for resectable rectal cancer. $N$ Engl J Med. 2001;345:638-46.

21. Yeo CJ, Abrams RA, Grochow LB, Sohn TA, Ord SE, Hruban $\mathrm{RH}$, et al. Pancreaticoduodenectomy for pancreatic adenocarcinoma: postoperative adjuvant chemoradiation improves survival. A prospective, single-institution experience. Ann Surg. 1997;225:621-33.

22. Corsini MM, Miller RC, Haddock MG, Donohue JH, Farnell MB, Nagorney DM, et al. Adjuvant radiotherapy and chemotherapy for pancreatic carcinoma: the Mayo Clinic experience (19752005). J Clin Oncol. 2008;26:3511-6.

23. Herman JM, Swartz MJ, Hsu CC, Winter J, Pawlik TM, Sugar E, et al. Analysis of fluorouracil-based adjuvant chemotherapy and radiation after pancreaticoduodenectomy for ductal adenocarcinoma of the pancreas: results of a large, prospectively collected database at the Johns Hopkins Hospital. J Clin Oncol. 2008;26:3503-10.

24. Regine WF, Winter KA, Abrams RA, Safran H, Hoffman JP, Konski A, et al. Fluorouracil vs. gemcitabine chemotherapy before and after fluorouracil-based chemoradiation following resection of pancreatic adenocarcinoma: a randomized controlled trial. JAMA. 2008;299:1019-26.

25. Reeves BC. Principles of research: limitations of non-randomized studies. Surgery (Oxford). 2008;26:120-4.

26. Klein JP, Moeschberger ML. Survival Analysis: Techniques for Censored and Truncated Data. New York: Springer-Verlag; 2003.

27. Cox DR, Oakes D. Analysis of Survival Data. London: Chapman and Hall; 1984.

28. Newgard CD, Hedges JR, Arthur M, Mullins RJ. Advanced statistics: the propensity score-a method for estimating treatment effect in observational research. Acad Emerg Med. 2004;11:953-61.

29. Further evidence of effective adjuvant combined radiation and chemotherapy following curative resection of pancreatic cancer. Gastrointestinal Tumor Study Group. Cancer. 1987;59:2006-10.

30. Evans DB, Varadhachary GR, Crane CH, Sun CC, Lee JE, Pisters $\mathrm{PW}$, et al. Preoperative gemcitabine-based chemoradiation for 
patients with resectable adenocarcinoma of the pancreatic head. J Clin Oncol. 2008;26:3496-502.

31. Varadhachary GR, Wolff RA, Crane CH, Sun CC, Lee JE, Pisters $\mathrm{PW}$, et al. Preoperative gemcitabine and cisplatin followed by gemcitabine-based chemoradiation for resectable adenocarcinoma of the pancreatic head. J Clin Oncol. 2008;26:3487-95.
32. Oettle H, Post S, Neuhaus P, Gellert K, Langrehr J, Ridwelski K, et al. Adjuvant chemotherapy with gemcitabine vs. observation in patients undergoing curative-intent resection of pancreatic cancer: a randomized controlled trial. JAMA. 2007;297:267-77. 